\title{
ON APPLICATIONS OF LITTLE'S FORMULA ${ }^{1}$
}

\author{
JEWGENI H. DSHALALOW \\ Department of Applied Mathematics \\ Florida Institute of Technology \\ Melbourne, FL 32901, USA
}

\begin{abstract}
In classical Little's formula $L=\lambda W$ used in queueing, the parameter $\lambda$ serves as the intensity of the input process. In various applications this parameter may not be known. The author discusses important classes of modulated input processes, where this parameter can be found.

Key words: intensity, modulated process, Cox process, nonhomogeneous Poisson process, semi-Markov process, semi-regenerative process, Little's formula, queueing process, waiting time process.
\end{abstract}

AMS Subject Classification: Primary $60 \mathrm{G} 57,60 \mathrm{~K} 15,60 \mathrm{~K} 99$, secondary $60 \mathrm{~K} 10,60 \mathrm{~K} 25$.

\section{INTRODUCTION}

Little's formula, $L=\lambda W$, belongs to one of the most distinguished and widely referred results in queueing. This formula connects the expected value, $L$, of the queue length and, $W$, waiting time processes (in their stochastic equilibrium) through $\lambda$, the total intensity of the input process. The total intensity of the input process is defined as the rate of incoming customers averaged over the infinite time horizon. More formally: let $\mathcal{H}=\left\{\tau_{n}, X_{n}\right\}$ be a marked point process (i.e. $\mathcal{H}=\sum_{i=1}^{\infty} X_{i} \varepsilon_{\tau_{i}}$, where $\varepsilon_{k}$ denotes the unit mass at point $k$ ). The total intensity of $\mathcal{H}$ is defined as $\lambda=\lim _{t \rightarrow \infty} \frac{1}{t} E[\mathcal{H}([0, t])]$. Specifically, for a Poisson input process with parameter $\lambda$ the total intensity coincides with this parameter, and for general recurrent-positive renewal processes $\lambda$ is the reciprocal of the mean inter-renewal time.

In many standard queueing systems studied in the past the total intensity was supposed to be either known as an input parameter or it could be obtained analytically, primarily for renewal processes. For various other processes the search for $\lambda$ can become a separate problem. Needless to say, the lack of information on $\lambda$

\footnotetext{
${ }^{1}$ Received: September, 1992. Revised: March, 1993.
} 
makes Little's formula impractical, since it is essential in evaluating $W$ with $L$ available or visa versa.

While it is virtually impossible to give a recipe for $\lambda$ in each case, we discuss some applications to queueing in the class of "modulated input processes." The most general scenario of such a process would be the one of a bulk arrival stream, formally represented as a marked random measure $\mathcal{H}=\sum_{i=1}^{\infty} X_{i} \varepsilon_{\tau_{i}}$, perturbed by an arbitrary (separable) jump process $\xi$ changing its values at random instants of time $T_{0}$, $T_{1}, \ldots$. The modulation assumes that over intervals $\left(T_{n}, T_{n+1}\right)$ input $\mathcal{H}$ will evolve "by itself" with no affect of $\xi$, but at each time point $T_{n}$, characteristics (such as probability distributions) of both $\tau_{i}$ and $X_{i}$ will depend on the value $\xi$ takes at $T_{n}$.

Markov-modulated Poisson processes form a very important special class of modulated processes arising in telecommunications and queueing [4]. In general, a Markov-modulated Poisson process is characterized by a pair of a nonstationary Poisson process $P$ with intensity $\lambda(t)$ and a jump Markov process $\mathcal{H}$ that makes this intensity $\lambda(\mathcal{H}(t))$. [An excellent survey of Markov-modulated Poisson processes and their applications to queueing may be found in Prabhu and Zhu [3]].

More general than Markov modulated Poisson processes are semi-Markov and semi-regenerative modulated Poisson process. They find a large variety of applications in networking, inventories, queuing, dams and stock markets.

In this paper the author restricts to an extension and discussion of some results from his earlier paper [2] on intensities for semi-Markov and semi-regenerative modulated processes and their applications to Little's formula.

\section{BACKGROUND}

The following definitions and results are due to Dshalalow [2].

\section{Definition 1.}

Let $\{\Omega, \mathcal{F}, P, \xi(t), t \in \mathbb{B}\} \rightarrow(\Psi, \mathfrak{B}(\Psi))$ be a stochastic jump process on $\&$ (where, in general, $\&$ is a locally compact and $\sigma$-compact topological space with a countable base), and let $\xi_{\omega}$ denote a $\omega$-section of $\xi$. Let $\bigcup_{j=1}^{\infty} D_{j}$ be at most countable, measurable decomposition of $\Psi$. Then $\bigcup_{j=1}^{\infty} B \cap \xi_{\omega}^{-1}\left(D_{j}\right)$ is a countable measurable decomposition of a Borel set $B \in \mathfrak{B}(\mathscr{\&})$. Consider a marked random measure 


$$
Z^{j}=\sum_{i} X_{i}^{(j)} \varepsilon_{\tau_{i}^{(j)}}
$$

with mark space $\mathcal{E}^{\prime}=[0, \infty]$. The random measure

$$
Z^{\xi}(B)=\sum_{j=0}^{\infty} Z^{j}\left(B \cap \xi_{\omega}^{-1}\left(D_{j}\right)\right)
$$

is called a marked random measure modulated by the process $\xi$.

Let $Z^{j}$ be a compound random measure with mark space $\mathcal{S}^{\prime}, Z^{j}=\sum_{i} X_{i}^{(j)} \varepsilon_{\tau_{i}^{(j)}}$ obtained from the underlying counting measure $N_{j}=\sum_{i} \varepsilon_{\tau_{i}(j)}$ by independent marking, and for each $j$ let $\left\{X_{i}^{(j)} ; i=1,2, \ldots\right\}$ be a sequence of independent and identically distributed random variables with common mean $\alpha_{j}$. Assume that $N_{j}$ is a Poisson counting measure directed by a random measure $\mathcal{H}_{j}$, i.e. $(N, \mathcal{H})=\left(N_{j}, \mathcal{H}_{j}\right)$ is a Cox process. The following is an extension of Lemma 3.2 in Dshalalow [2].

Theorem 2. For the random measure $Z^{\xi}$ modulated by the Cox process $(N, M)$ and with the initial measure $\nu$,

$$
E^{\nu}\left[Z^{\xi}\right]=\sum_{j=0}^{\infty} \alpha_{j} E^{\nu}\left[\int_{B} P^{\nu}\left\{\xi(u) \in D_{j}\right\} \mathcal{H}_{j}(d u)\right]
$$

where $E^{\nu}$ denotes the expected value with respect to probability measure $P^{\nu}$.

Let $\&=\mathbb{R}, \mathcal{M}_{j}$ be a nonrandom absolutely continuous (with respect to the Lebesgue measure $\mathcal{L}$ ) Borel measure, and $\gamma_{j}$ be its Radon-Nikodym density. Then, from Theorem 2 we have

$$
E^{\nu}\left[Z^{\xi}\right]=\sum_{j=0}^{\infty} \alpha_{j} \int_{B} \gamma_{j}(u) P^{\nu}\left\{\xi(u) \in D_{j}\right\} \mathcal{L}(d u) .
$$

In particular, if for each $j$, the Poisson counting measure $N_{j}$ is stationary, i.e. if $\gamma_{j}=\lambda_{j}$ (a positive constant), we have:

$$
E^{\nu}\left[Z^{\xi}\right]=\sum_{j=0}^{\infty} \alpha_{j} \lambda_{j} \int_{B} P^{\nu}\left\{\xi(u) \in D_{j}\right\} \mathcal{L}(d u)
$$

Definitions 3. Throughout the remainder of this paper we will consider the Borel $\sigma$-algebra generated by the usual topology on the real line.

(i) For every Borel set $B$ and every Lebesgue-Stieltjes measure $\mathcal{L}_{s}$ the number

$$
\lambda_{B}=\frac{E^{x}\left[Z^{\xi}(B)\right]}{\ell_{s}(B)} \in[0, \infty]
$$

is called the total intensity of $Z^{\xi}$ (over the set $B$ with respect to $\mathcal{L}_{s}$ ).

(ii) If $\mathcal{L}$ is the restriction of the Lebesgue measure on $\mathfrak{B}\left(\mathbb{R}_{+}\right)$and $\left\{B_{t} ; t \in \mathcal{N}\right\}$ is a monotone increasing family of sets along a net $\mathcal{N}$ such that $\cup_{t \epsilon} \mathcal{N} B_{t}=\mathbb{R}_{+}$, we call the number

$$
\lambda=\lim _{t \in \mathcal{N}} \frac{E^{x}\left[Z^{\xi}\left(B_{t}\right)\right]}{\mathcal{L}\left(B_{t}\right)} \in[0, \infty],
$$


the total intensity of $Z^{\xi}$ over $\mathbb{R}_{+}$.

\section{APPLICATIONS}

\section{LITTLE'S FORMULA FOR QUEUES WITH INPUTS MODULATED BY SEMI-MARKOV PROCESSES.}

Definitions 4. (Dshalalow [2].)

(i) Let $\mathcal{H}=\sum_{i=0}^{\infty} \xi_{i} \varepsilon_{T_{i}}$ be an irreducible and aperiodic Markov renewal process with a discrete state space $\Psi$ for $\xi_{n}$. Denote $\beta_{x}=E^{x}\left[T_{1}\right]$ and $\beta=\left(\beta_{x} ; x \in \Psi\right)$. Suppose that the embedded Markov chain $\xi_{n}$ is ergodic and that $P=\left(p_{x} ; x \in \Psi\right)$ is its invariant probability measure. $\mathcal{H}$ is called recurrent-positive if its mean interrenewal time, $\boldsymbol{P} \boldsymbol{\beta}^{\mathrm{T}}$, is finite. An irreducible and aperiodic and recurrent-positive Markov renewal process is called ergodic.

(ii) Let $\&=\mathbb{R}_{+}$and let $N_{j}$ be a stationary orderly Poisson counting measure with intensity $\lambda_{j}$. For each $j$, suppose that $\left\{X_{i}^{(j)}\right\}$ is a sequence of nonnegative integer-valued random variables with finite means $\alpha_{j}$. Let $\left\{\Omega, \mathcal{F},\left(P^{x}\right)_{x \in \Psi}, \xi(t), t \geq 0\right\}$ $\rightarrow \Psi=\{0,1, \ldots\}$ be the minimal semi-Markov process associated with a Markov renewal process $\mathcal{H}=\left\{\Omega, \mathcal{F},\left(P^{x}\right)_{x \in \Psi}, \xi_{n}=\xi\left(T_{n}+0\right), T_{n}\right\} \rightarrow\left(\Psi \times \mathbb{R}_{+}, \mathfrak{B}\left(\Psi \times \mathbb{R}_{+}\right)\right)$, where $P^{x}=P^{\epsilon}$. Then, $Z^{\xi}$ in (1) is called a marked Poisson random measure modulated by the semi-Markov process $\xi$.

Theorem 5. Let $\mathcal{H}$ be an ergodic Markov renewal process and let $Z^{\xi}$ a marked Poisson random measure modulated by the associated semi-Markov process $\xi$. Denote $\beta=\left(\beta_{x} ; x \in \Psi\right), \quad \lambda=\left(\lambda_{x} ; x \in \Psi\right), \quad \alpha=\left(\alpha_{x} ; x \in \Psi\right)$ and $\rho=\alpha * \beta * \lambda$, (the Hadamard product of vectors $\alpha, \beta$ and $\lambda$ ). Then, in a queueing system with the input $Z^{\xi}$, the following version of Little's formula holds true:

$$
L=\frac{P \rho^{\mathrm{T}}}{P \beta^{\mathrm{T}}} W .
$$

Proof. For some Borel set $B$ denote

$$
\Theta^{x}(j, B)=\int_{B} P^{x}\{\xi(u)=j\} \mathcal{L}(d u)
$$

and

$$
\Theta^{x}(B)=\left(\Theta^{x}(j, B) ; j \in \Psi\right)
$$


Then, since $\xi$ is ergodic, for all $x \in \mathcal{B}, \lim _{t \rightarrow \infty} \frac{1}{t} \Theta^{x}([0, t])$ exists; it is independent of $x$ and it is equal to $\frac{P * \beta}{P \beta^{T}}$. (For a reference, see Çinlar [1].) This and formula (2) yield that

$$
\lambda=\lim _{t \rightarrow \infty} \frac{1}{t} E\left[Z^{\xi}([0, t])\right]=\frac{P \rho^{\mathrm{T}}}{P \beta^{\mathrm{T}}} .
$$

for the total intensity $\lambda$ of the input $Z^{\xi}$ modulated by the semi-Markov process $\xi$.

\section{LITTLE'S FORMULA FOR QUEUES WITH INPUTS MODULATED BY SEMI-REGENERATIVE PROCESSES}

Let $Z^{\xi}$ be a marked random measure modulated by an ergodic semiregenerative process $\left\{\Omega, \mathcal{F},\left(P^{x}\right)_{x \in \Psi}, \xi(t) ; t \geq 0\right\} \rightarrow \Psi$ (relative to the Markov renewal process $\left.\left\{\xi_{n}, T_{n}\right\}\right)$ with the stationary probability distribution $\pi=\left(\pi_{j} ; j \in \Psi\right)$.

Theorem 6. In a stochastic system with the input $Z^{\xi}$ the following version of Little's formula for semi-regenerative modulated Poisson process holds true:

$$
L=\pi(\lambda * \alpha) W .
$$

The statement of the theorem is due the result in Dshalalow [2]: the semiregenerative modulated Poisson process $Z^{\xi}$ has the total intensity

$$
\lambda=\lim _{t \rightarrow \infty} \frac{1}{t} E^{\alpha}\left[Z^{\xi}([0, t])\right]=\pi(\lambda * \alpha) .
$$

Acknowledgement. The implementation of referee's helpful suggestions and criticism considerably improved the readability of the paper.

\section{REFERENCES}

[1] Çinlar, E., Markov renewal theory, Adv. Appl. Prob., 1, 123-187, 1969.

[2] Dshalalow, J., On modulated random measures, JAMSA, 4, No. 4, 305-312, 1991.

[3] Prabhu, N.U. and Zhu, Y., Markov-modulated queueing systems, Queueing Syst., 5, 215246, 1989.

[4] Stavrakakis, I., Queueing analysis of a class of star-interconnected networks under Markov modulated output process modeling, IEEE Trans. Comm., 40, No. 8, 1345-1357, 1992. 


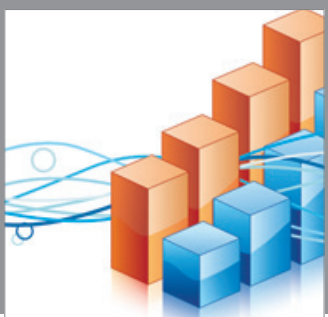

Advances in

Operations Research

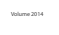

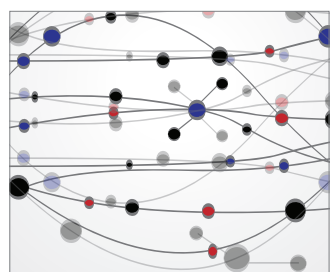

\section{The Scientific} World Journal
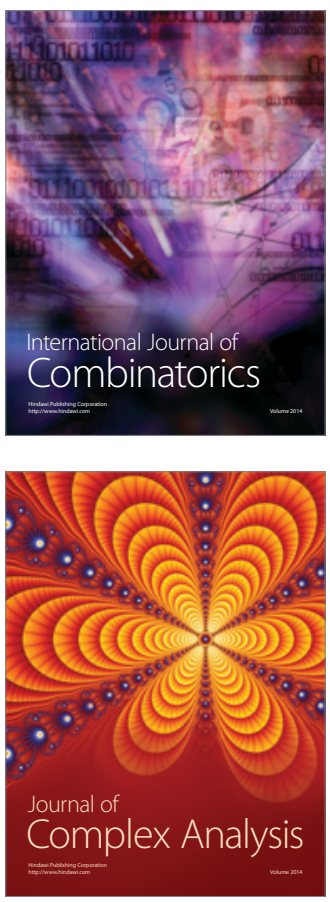

International Journal of

Mathematics and

Mathematical

Sciences
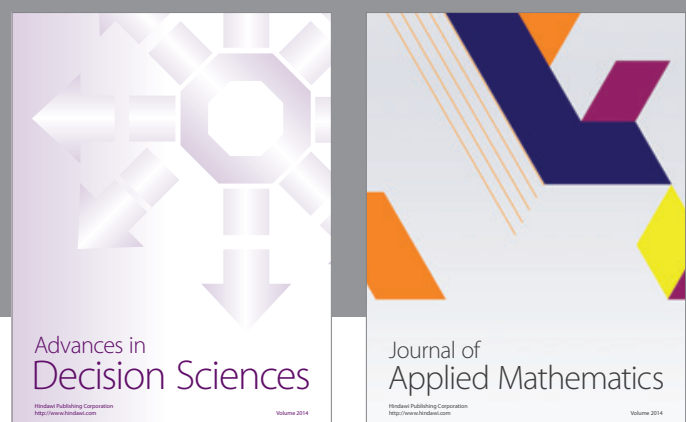

Journal of

Applied Mathematics
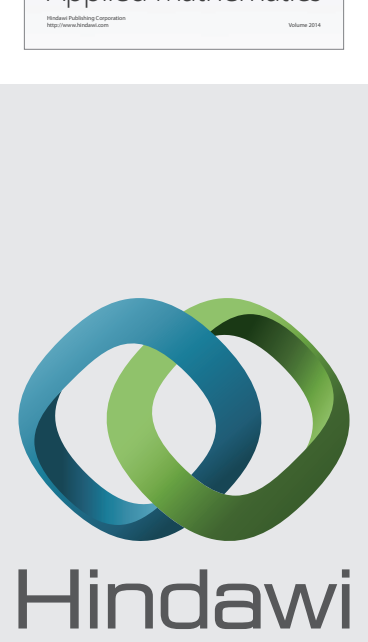

Submit your manuscripts at http://www.hindawi.com
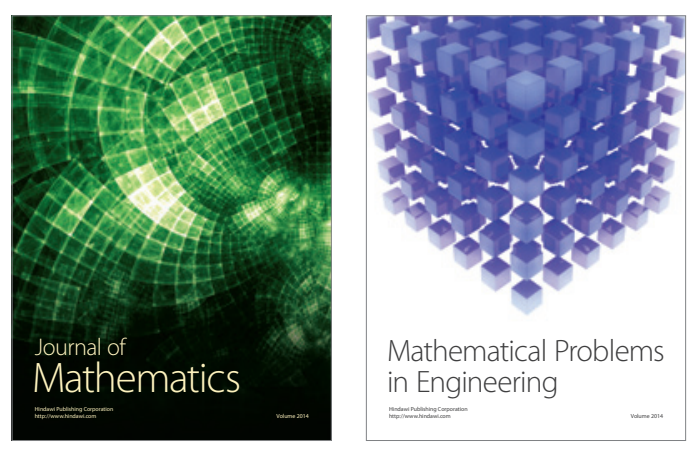

Mathematical Problems in Engineering
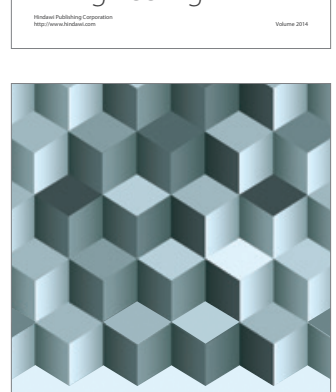

Journal of

Function Spaces
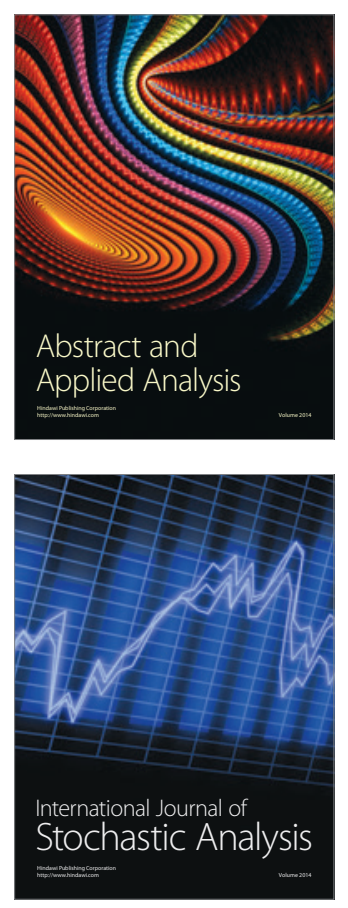

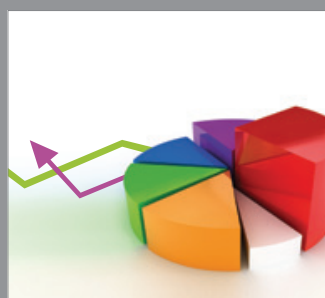

ournal of

Probability and Statistics

Promensencen
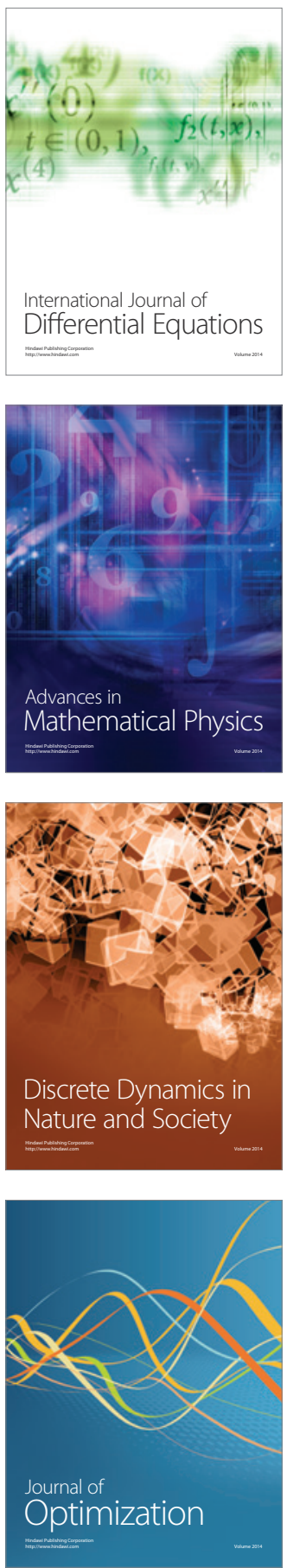